\title{
Impact of Performance Based Financing on Health Facilities Performance in Immunization in Two Health Districts in Benin
}

\author{
Agonnoude Togbédji Maurice ${ }^{1, *}$, Houeto Sègbegnon David ${ }^{1}$, Sambieni N'koué Emmanuel $^{2}$ \\ Adoukonou Tododjitché Thierry Armel ${ }^{1,3}$ \\ ${ }^{1}$ National School of Epidemiology and Public Health (ÉNATSE), University of Parakou, Parakou, Benin \\ ${ }^{2}$ Letters, Arts and Human Sciences Faculty (FLASH), University of Parakou, Parakou, Benin \\ ${ }^{3}$ Faculty of Medicine, University of Parakou, Parakou, Benin
}

Email address:

amaurte@yahoo.fr (A. T. Maurice), dhoueto@gmail.com (H. S. David), esambieni@yahoo.fr (S. N. Emmanuel), adoukonouthierry@yahoo.fr (A. T. T. Armel)

*Corresponding author

\section{To cite this article:}

Agonnoude Togbédji Maurice, Houeto Sègbegnon David, Sambieni N'koué Emmanuel, Adoukonou Tododjitché Thierry Armel. Impact of Performance Based Financing on Health Facilities Performance in Immunization in Two Health Districts in Benin. Central African Journal of Public Health. Vol. 6, No. 5, 2020, pp. 293-298. doi: 10.11648/j.cajph.20200605.18

Received: September 3, 2020; Accepted: September 22, 2020; Published: October 7, 2020

\begin{abstract}
Background: two pilot studies of RBF have been conducted in some health districts of Benin since 2012, which results have been scaled up in 2015. Then, it's important to see whether the impacts of health care from those studies will be confirmed by HFs performance in IS. Objective: to analyze the impact of RBF on IS performance in Parakou/N'dali and Nikki/Kalalé/Pèrèrè health districts, through the quality of the structure and the process of supplying these services, and their possible association with the immunization services performance. Method: A retrospective study was conducted including all public and private functional HFs covered by the period of 14 trimesters (T1 2014 to T2 2017). The dependent variable was the performance in IS. The independent variables were the quality of the structure, the quality of the process and the environment. A linear regression model was adjusted; the significance threshold was 5\%. Results: 55 HFs participated to the study; they were mostly in rural areas (76.4\%) and 36.4\% were at the first level of the pyramid. There was no improvement in both trained staff $(\mathrm{p}=0.67)$ and midwifery staff $(\mathrm{p}=0.75)$ and in the rest of the structure $(\mathrm{p}=0.697)$; however there was a significant improvement in supplying process of immunization services $(\mathrm{p}=0.001)$ and the performance in IS $(\mathrm{p}=0.010)$. In multivariate analysis, overall and in cluster by area analysis, models were not significant. Conclusion: RBF act only on the processes of supplying immunization services to improve HF performance, taking into account the environment. No explained factors were found for this performance improvement. Local dynamics in each HF should be in action which can be uncover in interactions with local actors.
\end{abstract}

Keywords: Immunization, Results Based Financing, Healthcare Quality, Structure, Process, Benin

\section{Introduction}

Performance based financing (PBF) is a mode of financing based on the assumption that linking motivation to performance would contribute to improving accessibility, quality and equity in the provision of health services [1]. In Benin, the RBF has made its debut in its practical phase since 2012 with two experiences: one funded by the World Bank in eight health zones $[2,3]$ and the second in five other health districts of the Departments of Mono-Couffo and Donga with the support of Belgian Technical Cooperation [4]. The basic idea was to strengthen the performance of the health system through essentially [2]: (i) the implementation of the contractual approach, (ii) the implementation of the process of improving the quality of health care and services and (iii) the introduction of financial incentives proportional to the 
production of care and services. The results of these different experiments were conclusive in terms of improving the quality of care, which led to the decision to expand to the other health districts of the country from June 2015. Since the conditions of the pilot experiment were never those of generalization, it was desirable two years after the start of this generalization, to see the impact of RBF on the performance of the FS of two sanitary zones of the Department of Borgou (the sanitary zones Parakou N'dali $(\mathrm{PN})$ and Nikki Kalalé Pèrèrè $(\mathrm{NKP})$ in terms of improving the quantity and quality of services offered to peoples.

The activities covered by this funding are part of the high impact intervention package (HPI) and relate to maternal and child protection activities and priority diseases (malaria, HIV/AIDS and tuberculosis). These activities are grouped into a minimum set of activities (MPA) for peripheral health training. Among the activities of maternal and child protection is the provision of iservices. Indeed, no personal, family or national development is possible without a reduction in child morbidity and mortality. Despite efforts to achieve immunization coverage, an estimated 21.8 million children worldwide are still unaffected by routine immunization services. These failures or delays in immunization of children in high-risk groups may limit the impact of these immunization programs on the burden of the disease [5].

In Benin, according to the preliminary report of the demographic and Health Survey 2017-2018 [6], the proportion of fully vaccinated children is $67.9 \%$. In the Department of Borgou, that of the 2 health zones covered by our study, this proportion is $54.2 \%$ (11th Department in Benin). That is why the PNDS 2018-2022 [7] of Benin has, to improve the quality of care and services, chosen as one of the axes of intervention the strengthening of the fight against diseases preventable by vaccination.

The objective of this study is to analyze the impact of RBF on the vaccine performance of FS in the PN and NKP health areas through the quality of the structure and process of offering these services and the possible link between the performance on the one hand, the quality of the process and structure on the other.

\section{Methods}

This is a retrospective longitudinal study aimed at assessing the influence of the structure, process and context of care production in the health formations of the Parakou-N'dali and Nikki-Kalalé-Pèrè health districts exposed to results-based funding on the performance of these health care organizations. A total of 55 functional FS from the beginning to the end of the experiment were included. The dependent variable (DV) was the number of fully vaccinated children. The main independent variables related to the structures (material, human and financial resources) to provide PF services as defined in the quality grid of RBF in Benin. The other independent explanatory variables concerned processes (specific training, supply planning, supervision, compliance with care procedures and protocols) and context (FS environment and level in the health pyramid). Data were collected using a routine data sheet (for the period 2014-2015) and quantitative and qualitative RBF data from Q3 2015 onwards. The rates of increase in the number of fully vaccinated children were calculated over the trimesters before and after the onset of BRF for comparison using the mean-matched comparison t-test. Similarly, the structural and process quality indicators before the start and towards the end of the experiment were calculated for an average comparison using the same procedure. Finally, a linear regression model using as a dependent variable the rate of increase in the number of fully vaccinated children over the duration of the experiment was adjusted. In our modeling strategy, we performed bi-varied analyses to detect independent variables with potential to influence RV as well as those that could be related to each other to avoid collinearities in the model. The significance threshold is $5 \%$.

\section{Results}

\subsection{Descriptive Characteristics}

Table 1 shows the repartition of health facilities according to contextual variables.

Table 1. Sample characteristic according to contextual Variables.

\begin{tabular}{lll}
\hline Variables and modalities & Frequency & \% \\
\cline { 2 - 3 } & $\mathbf{N}$ & 76.4 \\
\hline Area of health facility & 55 & 23.6 \\
Rural & 42 & 36.4 \\
Semi-urban or urban & 13 & 43.6 \\
Health facility's level on the pyramid & & 20 \\
Isolated dispensary or Maternity & 24 & 20.0 \\
HF of municipal borough & 11 & \\
Commune or private Health Centres & & \\
\hline
\end{tabular}

More than $3 / 4$ of the health facilities were located in rural areas and $7 / 10$ of them were isolated dispensary or maternity hospitals or district health centres.

\subsection{Performance in Vaccination}

Figure 1 shows the evolution of the number of fully vaccinated children in the Parakou N'dali (PN) and Nikki Kalalé Pèrèrè (NKP) health districts. There is a trend in saw tooth at the level of both areas with an overall downward trend as indicated by the trend lines. Tables 2 and 3 show the evolution of Human Resources, structure and process at the HF level of the two health districts. 


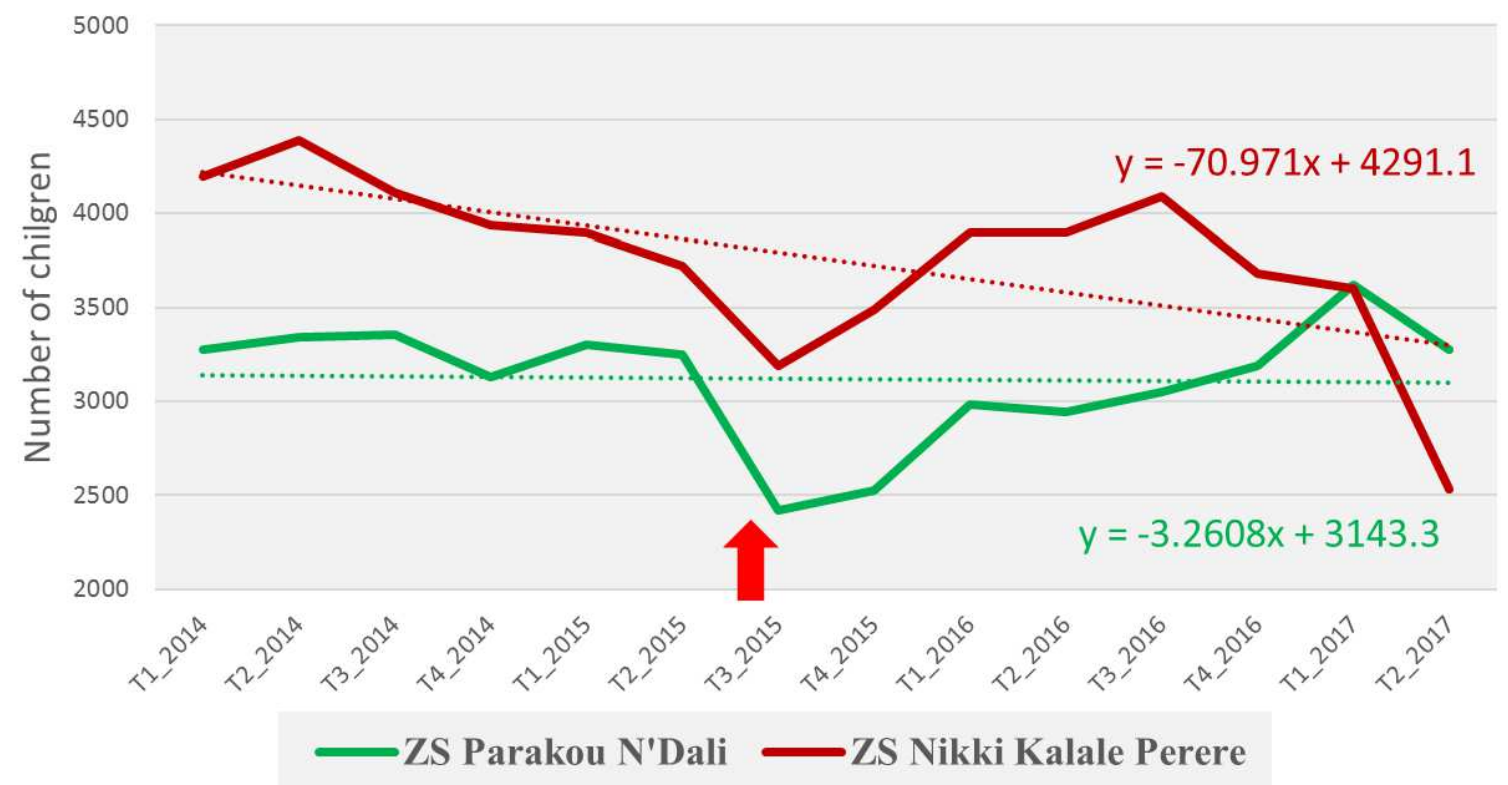

Figure 1. Evolution of number of children fully immunised in each health districts from T1 2014 to T2 2017.

The red arrow indicates the start of the PBF experiences in the two health districts.

Table 2. Evolution of human resources in health facilities of $P N$ and $N K P$ health districts before and after PBF.

\begin{tabular}{|c|c|c|c|c|}
\hline Variables et modalities & $\mathbf{N}$ & $\%$ & OR $95 \%$ CI & p-value \\
\hline \multicolumn{5}{|c|}{ Adequacy of qualified personnel according to WHO standards $=$ Yes } \\
\hline At the end of PBF & 14 & 25.5 & \multirow{2}{*}{$0.88[0.47-1.61]$} & \multirow{2}{*}{0.67} \\
\hline Before the PBF & 16 & 29.1 & & \\
\hline \multicolumn{5}{|c|}{ Adequacy of midwifery staff according to WHO standards $=$ Yes } \\
\hline At the end of PBF & 6 & 10.9 & \multirow{2}{*}{$1.2[0.39-3.7]$} & \multirow{2}{*}{0.75} \\
\hline Before PBF & 5 & 9.1 & & \\
\hline
\end{tabular}

There was no significant difference in the evolution of either skilled personnel $(p=0.67)$ or midwifery personnel $(p=0.75)$ between before and after RBF.

Table 3. Evolution of Structure, process and performance for immunization in the Parakou-Ndali and Nikki-Kalalé Pèrèrè Health facilities before and at the end of $P B F$.

\begin{tabular}{|c|c|c|c|}
\hline \multirow{2}{*}{ Variables } & \multicolumn{2}{|c|}{ Mean \pm standard deviation } & \multirow{2}{*}{ T-Test (p-value) } \\
\hline & Before PBF & At the end of PBF & \\
\hline Structure for immunization & $0.51 \pm 0.32$ & $0.50 \pm 0.32$ & 0.697 \\
\hline Process pour immunization & $0.63 \pm 0.20$ & $0.73 \pm 0.22$ & $0.001 * * *$ \\
\hline Performance for immunization & $-4.80 \pm 9.36$ & $0.03 \pm 11.44$ & $0.01 * *$ \\
\hline
\end{tabular}

$* *$ p significant at the threshold of $0.05 ; * * *$ p significant at the threshold of 0.001

It is noted that there is an improvement in the overall vaccination process and performance for immunization services in both areas.

\subsection{Associated Factors to Performance in Immunization}

For the multi-varied analyses purposes, we created three variables: structure evolution (difference between the structure index for $\mathrm{PF}$ at the end of FBR and the structure index at the beginning), process evolution (difference between the process Index at the end of FBR and the process Index at the beginning) and overall vaccination performance (growth rate of the number of fully vaccinated children over the entire study period).

Table 4. Factors associated to Immunization performance: not standardizes A coefficient in univariate analysis.

\begin{tabular}{|c|c|c|c|c|}
\hline Variables & $\mathbf{n}$ & Coefficient non standardisé A & $95 \%$ CI & p-value \\
\hline Evolution of the structure & \multirow{2}{*}{55} & 0.094 & {$[-5.71 ; 5.90]$} & 0.974 \\
\hline Evolution of the process & & -0.35 & {$[-8.13 ; 7.42]$} & 0.928 \\
\hline
\end{tabular}


Moreover, the correlation analysis between these two variables showed that they are strongly correlated with each other $(\rho=0.359, p=0.007)$. For this, in the multi varied model, we only consider the evolution of the process.

Univariate analysis showed that neither structure nor process changes influenced immunization performance of health facilities. In multi-varied analysis, globally as using the medium variable as a filter, neither the evolution of the process let alone the interaction variable influenced the performance of the FS.

Table 5. Facteurs associés à la performance en vaccination: coefficients non standardisés en analyse multi variée.

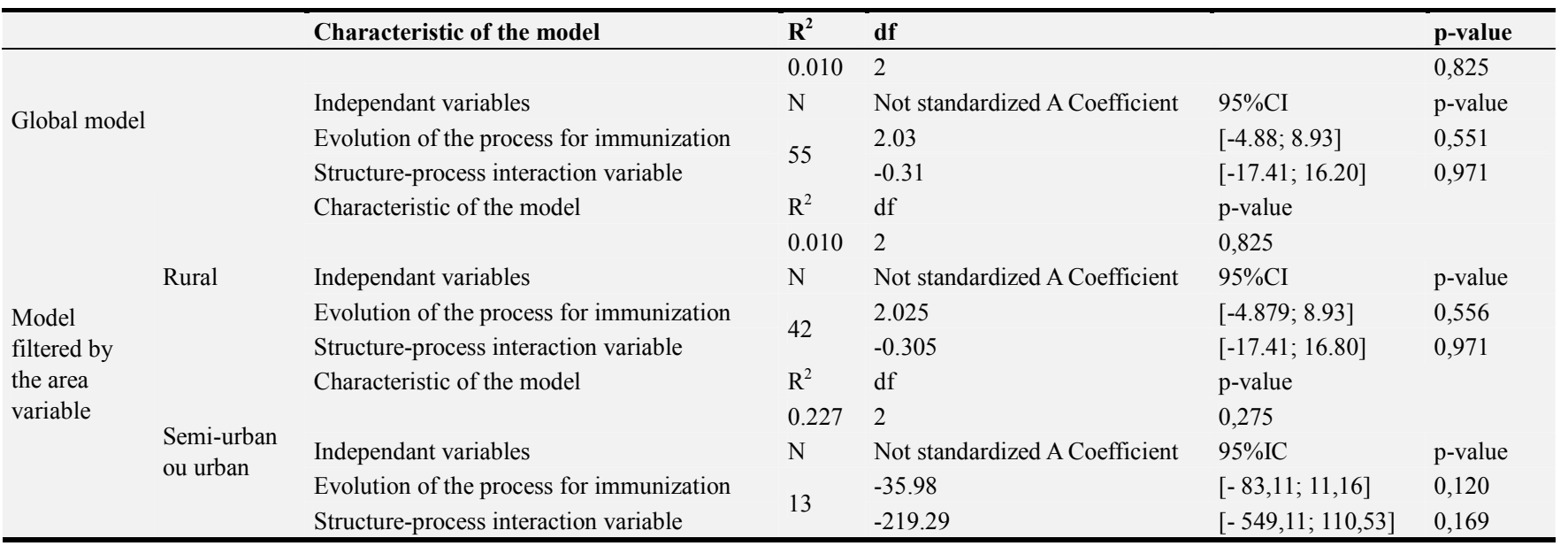

\section{Discussion}

This study of the influence of RBF on the vaccination performance of FS in PN and NKP areas was based on a longitudinal estimate with repeated experimentation with strong internal validity. However, a number of elements invite us to relativize the scope of our conclusions. First, we had to use secondary RBF data even though they were supplemented as far as possible by data directly collected in the field. Second, the study focused mainly on management data without the input of either health workers or clients for triangulation; this data was collected in the research project but is reserved for further exploitation. Despite these limitations, this study has led to conclusions that are not lacking in interest. It allows us to address three main issues.

Has the RBF had an impact on improving the quality of immunization services? In contrast to current studies on the issue, the quality of services in this study was divided into two sub-components, namely the quality of the structure and the quality of the process. The answer to the question is nuanced: yes there was an improvement in the process for vaccination but there was no improvement in the quality of the structure (excluding human resources) for the FS of both areas. As Paul et al. [4] pointed out, the Benin RBF, model BM as that of the CTB have not had a direct effect in terms of improving human resources. This effect on Human Resources was indirect and concerned ongoing support and training in the management and monitoring of activities and their motivation from various sources through the RBF instruments. But given the importance of human resources in the smooth running of FS activities, one wonders whether it would not have been more sensible for the state to take the initiative to qualitatively and quantitatively strengthen the staff before the start of the RBF to better achieve the results of the experiment. The opinion of the interested parties may be very informative to this effect. On the contrary, the improvement of the vaccination process seems logical since the project activities are mainly oriented towards the training of agents and their follow-up in the direction of continuous improvement of their procedures and modes of operation.

The overall improvement in the quality of services (structures and processes) is found in several studies: Rudasingwa et al. [8], in Burundi in terms of family planning, the overall quality during the mid-term evaluation of the pilot projects financed by the WB in Cameroon and Benin [9].

Has the RBF had an impact on improving the performance of FS in immunization services? The answer to this second question is probably yes: there was indeed an improvement in immunization performance (fully vaccinated children) for the health facilities of the two health zones over the period of study. This finding is also consistent with that made by several authors such as Ndikubagenzi et coll [10] in Burundi, Bonfrer and coll [11], Mussah and coll [12] in Liberia. Indeed, Ndikubagenzi and coll [10] found a statistically significant increase in the average increase in female users of PF services between the pre-scaling and post-scaling period of RBF in that country. Similarly, Bonfrer et al [11] noted a $4 \%$ improvement in the likelihood of a child being fully vaccinated with a more pronounced effect in the poorest. In Liberia, Mussah et al. [12] found that, compared to regions not supported by a performance-based funding project, regions supported by such a project experienced less deterioration in key maternal and child health indicators during the Ebola epidemic and a better improvement in these indicators in the post-Ebola period. On the contrary, no significant impact has been found on clinical productivity in Benin [9] and Eldridge and Palmer [13] have not found clear evidence on the effects of any type of RBF in 
the health context of any low-income country. These authors linked this result to the methodological weakness of most studies. Similarly, for Blacklock et al [14] in their systematic review of the issue, the impact of RBF on the performance of FS in PF is mixed: some studies showed an influence, others showed none. These authors rely on the cost of user services to explain this finding. In Benin, as immunization services for children are heavily subsidized, this mechanism seems to have failed.

How can one explain the improvement in the performance of FS in vaccination in the context of RBF in these two health zones of the Department of Borgou? Overall, as with the use of the medium variable as a filter, neither the evolution of the structure nor that of the process was an explanation for the improvement in vaccination performance. This finding disagrees with the work of Donabedian [15] emphasizes the importance of using not the overall quality of services in the analysis of RBF results but its structure and process components providing a small detail in the analysis of this information, the structure being the set of resources, instruments and technologies necessary for the production of care and the process related to the application of the best possible procedures for this production. Indeed, in this study, while the structure has not improved, the process, which in fact is related to the structure, has experienced an improvement but the link of this improvement with that of performance is not established.

\section{Conclusion: Implications for Actions}

The first important action to improve the performance of health training in the context of RBF should focus on both structures (workforce improvement) and processes (training supervision, promotion and control of standards of care). The lack of improvement in the number of staff in health training was the assumed choice of RBF experiences in Benin [2, 4] which clearly limited the scope of their results in terms of performance. The improvement of the process was a concern of these Beninese experiences of the RBF, which is proven; but given the increase in the workload that this calls for, if there is not a concomitant improvement in the number of staff, its effect became imperceptible or even reduced to naught. Taking these elements into account can have a sufficient impact on the overall quality likely to impact patient satisfaction and then performance in terms of the use of Health services in Benin

\section{Conflict of Interest}

The authors declare that they have no competing interests.

\section{Acknowledgements}

The authors acknowledge the Scientifical Board of Parakou University (Conseil Scientifique de l'Université de Parakou) for the 2016 Competitive Grant to principal researcher to perform this study. We thank all Health facilities managers and Health District managers in the two Health Districts who agreed to participate in this work.

\section{References}

[1] Canavan A, Toonen J, Elovainio R. Performance Based Financing An international review of the literature. Mauritskade 631092 AD Amsterdam: KIT Development Policy \& Practice, 2008.

[2] Projet de renforcement de la performance du système de santé (PRPSS). Document de cadrage du financement basé sur les résultats (FBR) au Bénin Version validée. PRPSS, MS. Cotonou: Programme de Renforcement de la Performance du Système de Santé, Ministère de la Santé, République du Bénin, 2014.

[3] Projet de renforcement de la performance du système de santé (PRPSS). Mise à échelle du FBR: pari gagné pour le Bénin: les 34 zones sanitaires couvertes à compter de juin 2015. Bulletin d'Information électronique du Projet de Renforcement de la Performance du Système de Santé, March 2015.

[4] Paul E, Dramé ML, Kashal J-P, et al. Performance-based financing to strengthen the health system in Benin: challenging the mainstream approach. Int J Health Policy Manag 2017; 6: $1-13$.

[5] Restrepo-Méndez MC, Barros AJ, Wong KL, et al. Inequalities in full immunization coverage: trends in low- and middle-income countries. Bull World Health Organ 2016; 2016 794-805A.

[6] République du Bénin. Cinquième Enquête Démographique et de Santé 2017-2018. Cotonou, Rockville, Maryland, USA: INSAE et ICF.: INSAE, MPD, Juillet 2018.

[7] République du Bénin, Ministère de la Santé. Plan national de développement sanitaire 2018-2022.

[8] Rudasingwa M, Soeters R, Basenya O. The effect of performance-based financing on maternal healthcare use in Burundi: a two-wave pooled cross-sectional analysis. Glob Health ACTION 2017; 10: 1-10.

[9] Fonds fiduciaire pour l'innovation en matière de résultats de santé (HRITF). Obtenir des résultats pour la santé des femmes et des enfants. Intermediary Report, New York: World Bank Group, 2015.

[10] Ndikubagenzi J, Nzeyimana É, Gahungere O, et al. Comparaison de l'utilisation des méthodes contraceptives modernes cinq ans avant et cinq ans après mise à échelle du financement basé sur la performance au Burundi. Bujumbura: Share. net, October 2017.

[11] Bonfrer I, Van de Poel E, Van Doorslaer E. The effects of performance incentives on the utilization and quality of maternal and child care in Burundi. Soc Sci Med 2014; 123: 96104.

[12] Mussah VG, Mapleh L, Ade S, et al. Performance-based financing contributes to the resilience of health services affected by the Liberian Ebola outbreak. Public Health Action 2017; 7: 100-105.

[13] Eldridge C, Palmer N. Performance-based payment: some reflections on the discourse, evidence and unanswered questions. Health Policy Plan 2009; 24: 160-166. 
[14] Blacklock C, MacPepple E, Kunutsor S, et al. Paying for Performance to Improve the Delivery and Uptake of Family Planning in Low and Middle Income Countries: A Systematic Review. Stud Fam Plann 2016; 47: 309-324.
[15] Donabedian A. Evaluating the Quality of Medical Care. Milbank Q 2005; 83: 691-729. 